\title{
Seleção de acessos de tomateiro resistentes à pinta-preta pela análise de agrupamento das curvas de progresso da doença
}

\author{
Bruno Soares Laurindo(1), Renata Dias Freitas Laurindo(1), Alcinei Místico Azevedo(1), Carlos Nick(1), \\ Derly José Henriques da Silva ${ }^{(1)}$ e Eduardo Seiti Gomide Mizubuti( ${ }^{(2)}$
}

\begin{abstract}
(1)Universidade Federal de Viçosa (UFV), Departamento de Fitotecnia, Avenida P. H. Rolfs, s/no, Campus Universitário, CEP 36570-900 Viçosa,MG,Brasil.E-mail:brunoslaurindo@yahoo.com.br, renataufv08@yahoo.com.br,alcineimistico@hotmail.com,carlos.nick@yahoo.com.br, derly@ufv.br (2)UFV, Departamento de Fitopatologia, CEP 36570-900 Viçosa, MG, Brasil. E-mail: mizubuti@ufv.br
\end{abstract}

\begin{abstract}
Resumo - O objetivo deste trabalho foi selecionar acessos resistentes à pinta-preta (Alternaria tomatophila) por meio da análise de agrupamento das curvas de progresso da doença em tomateiro (Solanum lycopersicum). Foram avaliados 134 acessos de tomateiro do Banco de Germoplasma de Hortaliças da Universidade Federal de Viçosa (BGH-UFV), no delineamento de blocos ao acaso, além das testemunhas suscetíveis 'Débora' e 'Santa Clara'. As plantas foram inoculadas com uma mistura de conídios de diferentes isolados de Alternaria spp. e avaliadas regularmente quanto à severidade da doença a cada três dias após a inoculação, no total de seis avaliações. Ajustou-se o modelo logístico aos dados de severidade da pinta-preta, e as estimativas obtidas para a incidência final da doença $\left(B_{1}\right)$ e a taxa de progresso da doença $\left(B_{3}\right)$ foram submetidas à análise de variância multivariada (Manova). As médias dessas estimativas, para cada acesso, foram submetidas à análise de agrupamento. Foram formados 24 grupos distintos com base no agrupamento das curvas de progresso da doença, o que possibilitou identificar os acessos BGH-2143, BGH-2235, BGH-2270 e BGH-2118 de tomateiro como potenciais fontes de resistência à pinta-preta.
\end{abstract}

Termos para indexação: Alternaria tomatophila, Solanum lycopersicum, banco de germoplasma, estatística multivariada, modelos não lineares, recursos genéticos.

\section{Selection of tomato accessions resistant to early blight by cluster analysis of disease progress curves}

\begin{abstract}
The objective of this work was to select accessions resistant to early blight (Alternaria tomatophila) through cluster analysis of the disease progress curves in tomato (Solanum lycopersicum). One hundred and thirty-four tomato accessions from the Banco de Germoplasma de Hortaliças of Universidade Federal de Viçosa (BGH-UFV) were evaluated in a randomized complete block design, as well as the susceptible controls 'Débora' and 'Santa Clara'. The plants were inoculated with a mixture of spores from different Alternaria spp. isolates and evaluated regularly regarding disease severity every three days after inoculation, totalizing six evaluations. The logistic model was adjusted to data on early blight severity, and the estimates obtained for final disease incidence $\left(B_{1}\right)$ and rate of disease progression $\left(B_{3}\right)$ were subjected to multivariate analysis of variance (Manova). The means of these estimates, for each access, were subjected to cluster analysis. Twenty-four distinct groups were formed based on disease-progress curve clusters, which allowed the identification of the BGH-2143, BGH-2235, BGH-2270, and BGH-2118 accessions as potential sources of resistance to early blight.
\end{abstract}

Index terms: Alternaria tomatophila, Solanum lycopersicum, germplasm bank, multivariate statistics, nonlinear models, genetic resources.

\section{Introdução}

O tomateiro (Solanum lycopersicum L.) é a segunda hortaliça em importância econômica no Brasil. Em 2011, a produção brasileira foi de aproximadamente 4,4 milhões de toneladas numa área colhida superior a 63 mil hectares, o que tornou o Brasil o nono colocado no ranking entre os principais países produtores (Quintana, 2013).
Apesar desse cenário favorável, o tomaticultor enfrenta grandes problemas que dificultam o manejo da cultura. Entre esses, destaca-se a pinta-preta, doença causada pelo fungo Alternaria tomatophila E.G. Simmons (Catão et al., 2013).

Como não há cultivares de tomateiro resistentes à doença (Grigolli et al., 2011), a medida mais utilizada para o controle da pinta-preta é a aplicação de fungicidas, o que acarreta o aumento do custo de

Pesq. agropec. bras., Brasília, v.50, n.2, p.106-114, fev. 2015

DOI: 10.1590/S0100-204X2015000200002 
produção e, também, de resíduos nos frutos (Vale et al., 2007). Uma solução para esse problema é o desenvolvimento de cultivares resistentes. Para isso, é necessário identificar fontes de resistência em acessos de tomateiro armazenados em bancos de germoplasma.

Geralmente, a seleção de genótipos resistentes é feita com base somente na área abaixo da curva de progresso da doença (Grigolli et al., 2011; Kumar \& Srivastava, 2013), de acordo com a metodologia algébrica proposta por Campbell \& Maden (1990). No entanto, o formato da curva de progresso da doença também apresenta informações importantes sobre a dinâmica da doença (Azevedo et al., 2012). Assim, esse critério também pode ser utilizado para a seleção dos melhores genótipos (Fiorini et al., 2010). Nessa situação, consideram-se como melhores os genótipos com menor área foliar lesionada nos últimos dias de avaliação e prioriza-se a seleção de genótipos cuja área foliar lesionada seja menor nos períodos iniciais de avaliação, o que pode proporcionar maior eficiência dos métodos de controle no período inicial do progresso da doença e reduzir riscos de danos à cultura.

Para a representação gráfica das curvas de progresso da doença, pode-se recorrer ao ajuste de modelos não lineares. Contudo, quando há muitos genótipos, a representação gráfica pode tornar-se inviável, por ser de difícil visualização (Azevedo et al., 2012). Nesse caso, o agrupamento das curvas por análise multivariada torna-se estratégia viável, uma vez que se agrupam curvas similares, o que facilita a representação gráfica e a seleção dos melhores genótipos, mesmo quando estes são avaliados em grande número (Fiorini et al., 2010). A utilidade dessa estratégia a torna alternativa para os estudos do progresso de doenças, como a requeima em tomateiro (Fiorini et al., 2010; Azevedo et al., 2012), das curvas de produção de café (Coffea canephora) (Cecon et al., 2008) e do acúmulo de matéria seca em plantas de alho (Allium sativum) (Puiatti et al., 2013).

O objetivo deste trabalho foi selecionar acessos resistentes à pinta-preta por meio da análise de agrupamento das curvas de progresso da doença em tomateiro.

\section{Material e Métodos}

Os experimentos foram conduzidos no campo experimental do setor de Olericultura da Universidade
Federal de Viçosa (UFV), em Viçosa, MG (2045'14"S, $42^{\circ} 52^{\prime} 53^{\prime \prime} \mathrm{W}$, a $648,74 \mathrm{~m}$ de altitude).

Foram avaliados 134 acessos de tomateiro do BGH-UFV, em três experimentos: 1, de outubro de 2009 a janeiro de 2010,33 acessos; 2 , de julho a outubro de 2010, 50 acessos; e 3, de setembro a dezembro de 2010, 51 acessos (Tabela 1). Nos três experimentos, foram utilizadas, como testemunhas suscetíveis à pinta-preta, as cultivares Débora e Santa Clara.

A semeadura foi realizada em bandejas de isopor de 128 células contendo substrato comercial para hortaliças. O transplantio foi feito para o campo quando as plantas apresentavam quatro folhas definitivas, no espaçamento de 1,0x0,5 m. Utilizou-se o delineamento de blocos ao acaso, com três repetições e cinco plantas por parcela, sendo a parcela útil constituída pelas três plantas centrais. As plantas foram conduzidas com uma única haste e tutoradas com fitilho na vertical. Os tratos culturais foram realizados de acordo com o recomendado para a cultura. A utilização de defensivos agrícolas foi suspensa 15 dias antes da inoculação e durante as avaliações para resistência à pinta-preta.

Quarenta e cinco dias após o transplantio, as plantas foram inoculadas com uma mistura de conídios de quatro isolados de Alternaria spp., patogênicos ao tomateiro, provenientes de diferentes regiões geográficas. Os isolados utilizados foram VBH2, As 129, As 149 e As 151, provenientes dos estados de Minas Gerais, Santa Catarina, Paraná e Rio Grande do Sul, respectivamente, da coleção de culturas do Laboratório de Biologia de Populações de Fitopatógenos, do Departamento de Fitopatologia, da UFV. Os isolados foram selecionados para incorporar o maior número de variantes de resistência qualitativa, para seleção de genótipos com resistência quantitativa.

A produção do inóculo foi realizada de acordo com a metodologia proposta por Cardoso (2010). Cultivaramse os isolados em meio $\mathrm{V} 8 \mathrm{CaCO}_{3}$ ágar $(175 \mathrm{~mL}$ de suco $\mathrm{V} 8,3 \mathrm{~g}$ de $\mathrm{CaCO}_{3}, 20 \mathrm{~g}$ de ágar e $1 \mathrm{~L}$ de água), a $25 \pm 2^{\circ} \mathrm{C}$, sob fotoperíodo de 12 horas. No quinto dia de incubação, adicionou-se água destilada a cada placa e, com um pincel, retirou-se o micélio. As placas foram mantidas sem tampa a $22 \pm 2^{\circ} \mathrm{C}$, sob seis lâmpadas de luz negra $40 \mathrm{~W}$ (Havells Sylvania Brasil Iluminação Ltda., São Paulo, SP), de 1,20 m de comprimento, distanciadas a $3 \mathrm{~cm}$ entre si e posicionadas a $30 \mathrm{~cm}$ de altura das placas, por 24 horas, em fotoperíodo de 16 horas de escuro e 8 horas de luz. Após esse período, 
Tabela 1. Acessos de tomateiro (Solanum lycopersicum) do Banco de Germoplasma de Hortaliças da Universidade Federal de Viçosa (BGH-UFV), avaliados em três experimentos ${ }^{(1)}$.

\begin{tabular}{|c|c|c|}
\hline Experimento 1 & Experimento 2 & Experimento 3 \\
\hline BGH-2153 & BGH-242 & BGH-2128 \\
\hline BGH-2157 & BGH-887 & BGH-2129 \\
\hline BGH-2165 & BGH-971 & BGH-2130 \\
\hline BGH-2175 & BGH-973 & BGH-2131 \\
\hline BGH-2177 & BGH-984 & BGH-2132 \\
\hline BGH-2178 & BGH-996 & BGH-2133 \\
\hline BGH-2179 & BGH-1025 & BGH-2134 \\
\hline BGH-2180 & BGH-1258 & BGH-2135 \\
\hline BGH-2181 & BGH-2009 & BGH-2136 \\
\hline BGH-2182 & BGH-2010 & BGH-2138 \\
\hline BGH-2183 & BGH-2011 & BGH-2141 \\
\hline BGH-2184 & BGH-2023 & BGH-2143 \\
\hline BGH-2186 & BGH-2025 & BGH-2144 \\
\hline BGH-2187 & BGH-2030 & BGH-2145 \\
\hline BGH-2188 & BGH-2040 & BGH-2146 \\
\hline BGH-2189 & BGH-2044 & BGH-2148 \\
\hline BGH-2192 & BGH-2045 & BGH-2149 \\
\hline BGH-2194 & BGH-2048 & BGH-2150 \\
\hline BGH-2196 & BGH-2049 & BGH-2151 \\
\hline BGH-2197 & BGH-2050 & BGH-2152 \\
\hline BGH-2198 & BGH-2051 & BGH-2158 \\
\hline BGH-2201 & BGH-2070 & BGH-2185 \\
\hline BGH-2206 & BGH-2087 & BGH-2235 \\
\hline BGH-2209 & BGH-2088 & BGH-2236 \\
\hline BGH-2212 & BGH-2089 & BGH-2243 \\
\hline BGH-2218 & BGH-2091 & BGH-2245 \\
\hline BGH-2220 & BGH-2093 & BGH-2247 \\
\hline BGH-2222 & BGH-2095 & BGH-2248 \\
\hline BGH-2226 & BGH-2096 & BGH-2249 \\
\hline BGH-2227 & BGH-2097 & BGH-2251 \\
\hline BGH-2230 & BGH-2098 & BGH-2252 \\
\hline BGH-2231 & BGH-2100 & BGH-2255 \\
\hline BGH-2233 & BGH-2105 & BGH-2259 \\
\hline- & BGH-2109 & BGH-2260 \\
\hline - & BGH-2110 & BGH-2261 \\
\hline - & BGH-2111 & BGH-2262 \\
\hline- & BGH-2112 & BGH-2264 \\
\hline- & BGH-2113 & BGH-2266 \\
\hline - & BGH-2114 & BGH-2267 \\
\hline - & BGH-2115 & BGH-2269 \\
\hline- & BGH-2116 & BGH-2270 \\
\hline - & BGH-2117 & BGH-2271 \\
\hline - & BGH-2118 & BGH-2273 (salada) \\
\hline - & BGH-2119 & BGH-2273 (periforme) \\
\hline - & BGH-2120 & BGH-2274 \\
\hline - & BGH-2121 & BGH-2275 \\
\hline - & BGH-2122 & BGH-2276 \\
\hline - & BGH-2123 & BGH-2277 \\
\hline - & BGH-2124 & BGH-2280 \\
\hline- & BGH-2125 & BGH-2281 \\
\hline - & - & BGH-2282 \\
\hline 'Débora' ${ }^{\prime(2)}$ & 'Débora' & 'Débora' \\
\hline 'Santa Clara'(2) & 'Santa Clara' & 'Santa Clara' \\
\hline
\end{tabular}

os conídios foram removidos com a adição de $10 \mathrm{~mL}$ de água destilada em cada placa, seguida de raspagem superficial da colônia com escova de cerdas macias. A suspensão foi filtrada em dupla camada de gaze esterilizada e recolhida em um béquer, e a concentração da suspensão foi ajustada para $2 \times 10^{3}$ conídios $\mathrm{mL}^{-1}$. Para manter a mesma representatividade de cada um dos quatro isolados, foi preparada uma suspensão de conídios com volumes iguais para cada isolado.

As inoculações das plantas nos três experimentos ocorreram em 10/1/2010, 6/10/2010 e 6/12/2010, respectivamente. As inoculações foram realizadas no período da tarde, por volta das $16 \mathrm{~h}$, com auxílio de pulverizador costal manual. Foram aplicados aproximadamente $10 \mathrm{~mL}$ de suspensão por planta, tendo-se ajustado o tempo de aplicação em função da vazão do bico do pulverizador.

Nos dias posteriores às inoculações, para garantir alta umidade ao ambiente, as plantas passaram a ser irrigadas por microaspersores três vezes ao dia, às 9 , 13 e $17 \mathrm{~h}$, por $20 \mathrm{~min}$. Três dias após as inoculações, iniciaram-se as avaliações quanto à severidade da pinta-preta. As avaliações dos experimentos foram realizadas entre 13 e 28 de janeiro de 2010,9 e 24 de outubro de 2010 e 9 e 24 de dezembro de 2010, respectivamente, em intervalos regulares de três dias, o que totalizou seis avaliações da severidade da doença em cada experimento.

Nas avaliações de severidade da doença, utilizouse o programa Severity PRO (Nutter, 1997) para o treinamento da equipe de avaliação, com o intuito de corrigir distorções inerentes à estimativa visual. No campo, dois avaliadores estimaram a severidade da doença sem o auxílio de escala diagramática, isto é, determinaram a percentagem do tecido vegetal afetado pela doença. Para a análise estatística, foi considerada a média das estimativas feitas pelos dois avaliadores para cada unidade experimental (parcela).

A análise dos dados de todos os acessos avaliados nos três experimentos foi feita em uma única análise estatística. Realizou-se, primeiramente, a análise de variância agrupada (análise conjunta) pelo esquema 2 apresentado por Cruz et al. (2014). Essa metodologia foi utilizada para ajustar os dados de cada experimento, o que ameniza a influência ambiental e permite que os tratamentos possam ser comparados entre experimentos. Segundo Cruz et al. (2014), essa estratégia é de grande interesse quando são conduzidos vários experimentos 
avaliando as testemunhas em comum, como é o caso do presente trabalho. Nessa situação, a correção dos dados é realizada em função do efeito ambiental, obtido pela média das testemunhas em cada experimento, subtraída da média geral das testemunhas, ao se considerar todos os experimentos. Conforme Pimentel-Gomes (2009), a análise conjunta por essa metodologia corresponde à análise em delineamentos de blocos incompletos, de grande flexibilidade, eficiência e simplicidade.

Para descrever o progresso da doença, foi processado o agrupamento de curvas por meio da análise multivariada, pela metodologia apresentada por Fiorini et al. (2010) e Azevedo et al. (2012). No estudo do progresso da doença, foram ajustados vários modelos, além do modelo exponencial dado por $\mathrm{y}=\mathrm{y}_{\mathrm{o}} \exp (\mathrm{rx})$, em que $\mathrm{y}_{\mathrm{o}}$ é a quantidade inicial da doença e r é a taxa de progresso da doença. Ajustaramse, também, modelos mais complexos, como os de Schnute, Mitscherlich, Richards, Gompertz, Meloun I, von Bertalanffy, Michaelis-Menten e o logístico, cujas funções são citadas por Silveira et al. (2011). A partir das médias do coeficiente de determinação $\left(\mathrm{R}^{2}\right)$ ajustado e do critério de informação de Akaike (AIC), selecionou-se o modelo mais adequado.

Foi realizada análise de variância multivariada com uso do programa Proc GLM (SAS Institute, 2002), com a opção análise de variância multivariada (Manova), para avaliar o efeito dos acessos do BGH-UFV, tendo-se considerado as estimativas dos parâmetros do modelo selecionado. As médias ajustadas por quadrados mínimos (LSMEANS) dessas estimativas foram extraídas e submetidas à análise de agrupamento no Proc Cluster (SAS Institute, 2002), pelo método centroide. Assim, foram obtidos os valores dos desvios-padrão da raiz do quadrado médio (RMSSTD), para a identificação do número ótimo de agrupamentos por meio do ajuste de um modelo de máxima curvatura. Além disso, utilizou-se o Proc Tree (SAS Institute, 2002) para visualizar o dendograma e verificar quais acessos pertencem aos diferentes grupos obtidos pela discriminação estatística.

Para a melhor interpretação dos resultados, calculou-se, para cada grupo, a partir dos acessos que o compuseram, a média e o desvio-padrão dos coeficientes ajustados pelos modelos e da área abaixo da curva de progresso da doença (AACPD), calculada por meio da expressão baseada em Campbell \& Madden (1990):

$$
\operatorname{AACD}=\left\{\sum_{\mathrm{i}=1}^{\mathrm{n}-1}\left[\left(\mathrm{y}_{\mathrm{i}}+\mathrm{y}_{\mathrm{i}+1}\right) / 2\right]\left(\mathrm{t}_{\mathrm{i}+1}-\mathrm{t}_{\mathrm{i}}\right)\right\},
$$

em que: $\mathrm{y}_{\mathrm{i}}$ e $\mathrm{y}_{\mathrm{i}+1}$ são a percentagem de área foliar lesionada observada na avaliação $i$ e na seguinte $i+1 ; t_{i}$ e $t_{i+1}$ são o intervalo de tempo (dias) entre as avaliações; e $\mathrm{n}$ é o número total de avaliações.

\section{Resultados e Discussão}

Os valores obtidos para $\mathrm{R}^{2}$ ajustado $(97,4 \%)$ e AIC $(12,276)$ mostraram melhor ajuste do modelo logístico aos dados de severidade, o que indica que este modelo é o mais adequado para descrever as curvas de progresso da pinta-preta nos acessos avaliados (Tabela 2). O modelo logístico também foi utilizado por Paula \& Oliveira (2003) no estudo do progresso da doença em tomateiro.

Uma vantagem em estudar doenças por meio de curvas de progresso com uso de modelos não lineares é o fato de os parâmetros apresentarem interpretação biológica (Maia et al., 2009). O uso desses coeficientes para o agrupamento de curvas por meio da análise multivariada tem-se mostrado eficiente no estudo do progresso de doenças (Fiorini et al., 2010; Azevedo et al., 2012), em especial, quando há número elevado de tratamentos (acessos), em que a apresentação das curvas em gráficos é inviabilizada.

$\mathrm{Na}$ análise de variância multivariada, foi constatado efeito significativo dos acessos sobre o vetor dos parâmetros $\mathrm{B}_{1}$ (média final da severidade da doença) e $\mathrm{B}_{3}$ (taxa de progresso). Isso indica que os genótipos

Tabela 2. Modelos utilizados para descrever as curvas de progresso da pinta-preta (Alternaria tomatophila) em tomateiro (Solanum lycopersicum) e respectivos avaliadores da qualidade do ajuste.

\begin{tabular}{lccc}
\hline Modelo & \multicolumn{3}{c}{ Qualidade do ajuste } \\
\cline { 2 - 4 } & $\mathrm{R}^{2}$ ajustado & AIC & Convergência (\%) $^{(1)}$ \\
\hline Schnute & 0,924 & 24,310 & 100,000 \\
Mitscherlich & 0,824 & 32,851 & 100,000 \\
Richards & 0,958 & 15,161 & 69,586 \\
Gompertz & 0,790 & 31,673 & 99,757 \\
Logístico & 0,974 & 12,276 & 96,404 \\
Meloun I & 0,824 & 32,856 & 100,000 \\
von Bertalanffy & 0,938 & 20,677 & 85,158 \\
Michaelis-Menten & 0,744 & 33,646 & 100,000 \\
Exponencial & 0,898 & 25,115 & 99,270 \\
\hline
\end{tabular}

${ }^{(1)} \mathrm{R}^{2}$, coeficiente de determinação; e AIC, critério de informação de Akaike. 
diferem entre si em relação aos parâmetros do modelo estatístico e que, consequentemente, o emprego da análise multivariada para a formação de grupos de genótipos similares quanto aos parâmetros do modelo é viável. Resultados semelhantes foram obtidos por Fiorini et al. (2010) e Azevedo et al. (2012).

Após a análise multivariada e a obtenção do dendograma, observou-se, a partir do comportamento da estatística RMSSTD (Figura 1), o número ótimo de grupos, pelo método da máxima curvatura. O RMSSTD mede a homogeneidade dos grupos formados (Liu et al., 2013) e possibilita a identificação de um número ótimo de agrupamentos pelo método da máxima curvatura (Cecon et al., 2008). O uso desse método é vantajoso, pois permite que o número de grupos definidos no dendograma não seja escolhido de forma subjetiva (Liu et al., 2013). Com essa técnica, foi possível reduzir as 136 curvas obtidas de cada um dos tratamentos para 24 grupos (Tabela 3), o que proporciona melhor visualização e interpretação dos dados. Ao utilizar essa
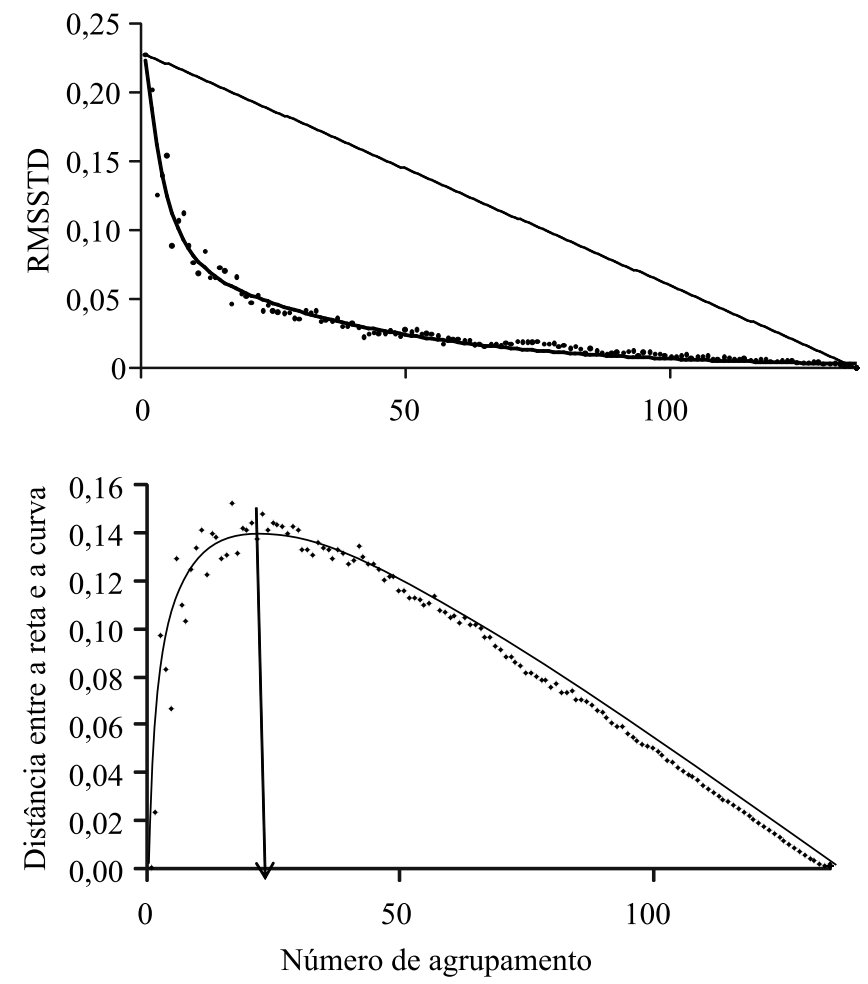

Figura 1. Comportamento da estatística desvio-padrão da raiz do quadrado médio (RMSSTD) e distância entre a reta e a curva estimada para verificação do número ótimo de agrupamento, em função do método da máxima curvatura.
Tabela 3. Acessos pertencentes aos grupos estabelecidos pelo corte no dendograma, tendo-se considerado o número ótimo de agrupamento em função do método da máxima curvatura, e número de acessos por agrupamento (№).

\begin{tabular}{lcc}
\hline $\begin{array}{l}\text { Agrupa- } \\
\text { mento }\end{array}$ & Acessos & № \\
\hline 1 & BGH-2087 & 1 \\
2 & BGH-2010, BGH-2030, BGH-2123, BGH-971 & 4 \\
3 & BGH-2048, BGH-2112, BGH-242 & 3 \\
4 & BGH-2113 & 1 \\
5 & BGH-2045, BGH-2051, BGH-2110, BGH-2111 & 4 \\
6 & BGH-2089, BGH-2276 & 2 \\
7 & BGH-2091 & 1 \\
8 & BGH-2098, BGH-2220, & 2 \\
9 & BGH-2230 & 1 \\
10 & BGH-2093, BGH-2243, BGH-2267 & 3
\end{tabular}

BGH-1258, BGH-2025, BGH-2088, BGH-2097, BGH-2100, BGH-2116, BGH-2121, BGH-2122, BGH-2135, BGH-2165, BGH-2187, BGH-2192, BGH-2196, BGH-2206, BGH-2233, BGH-2249

BGH-2120, BGH-2125, BGH-2141, BGH-2153, BGH-2177, BGH-2178, BGH-2186, BGH-2188, BGH-2226, BGH-2231, BGH-2236, BGH-2274, BGH-2282

BGH-2114, BGH-2130, BGH-2136, BGH-2144, BGH-2150, BGH-2151, BGH-2157, BGH-2175, 13 BGH-2180, BGH-2182, BGH-2184, BGH-2194, BGH-2197, BGH-2198, BGH-2218, BGH-2222, BGH-2227, BGH-2259, BGH-2281

BGH-2273 (salada), BGH-2011, BGH-2247

BGH-2260, BGH-2266,

BGH-2040, BGH-2105, Santa Clara

BGH-2049, BGH-2148, BGH-2255

BGH-2273 (periforme), BGH-1025, BGH-2023, BGH-2070, BGH-2096, BGH-2109, BGH-2115, BGH-2117, BGH-2119, BGH-2124, BGH-2131, BGH-2133, BGH-2134, BGH-2146, BGH-2149, BGH-2152, BGH-2158, BGH-2179, BGH-2189, BGH-2245, BGH-2251, BGH-2262, BGH-2269, BGH-2271, BGH-2275, BGH-2277, BGH-2280, BGH-996
19
BGH-2009, BGH-2132

BGH-984

BGH-2044, BGH-2050, BGH-2095, BGH-2128, BGH-2129, BGH-2145, BGH-2185, BGH-2248, BGH-2252, BGH-2261, BGH-2264, BGH-887, BGH-973, Débora

BGH-2138, BGH-2181, BGH-2183, BGH-2201, BGH-2209, BGH-2212

BGH-2143, BGH-2235, BGH-2270 BGH-2118 
mesma técnica, Azevedo et al. (2012) explicaram o comportamento de 42 acessos, que foram divididos em seis grupos, enquanto Fiorini et al. (2010) explicaram o comportamento de 27 acessos, em oito grupos. Maia et al. (2009) verificaram que o método de agrupamento multivariado tem grande potencial na comparação de curvas e é uma estratégia mais viável que os testes de identidade de modelo, nos casos em que são avaliados muitos tratamentos.

Para cada grupo formado, foi calculada a média e o desvio-padrão de $\mathrm{B}_{1}, \mathrm{~B}_{3}$ e AACPD dos tratamentos que compuseram os grupos (Tabela 4). Constataramse, para os grupos 1, 2 e 3, formados pelos acessos BGH-2087, BGH-2010, BGH-2030, BGH-2123, BGH-971, BGH-2048, BGH-2112 e BGH-242, elevados valores de $\mathrm{B}_{3}$, maior AACPD (Tabela 4) e maior severidade da doença (Figura 2); esses acessos foram considerados os mais susceptíveis.

Quanto às cultivares, Santa Clara fez parte do grupo 16, e Débora fez parte do grupo 21 (Tabela 3).
Estes dois grupos apresentam padrão intermediário em relação aos demais acessos avaliados quanto à resistência à pinta-preta (Figura 2 e Tabela 4). Grigolli et al. (2011), ao avaliar a resistência à pinta-preta, também encontraram acessos mais susceptíveis que as cultivares Débora e Santa Clara.

Entre os acessos com os maiores níveis de resistência à pinta-preta, destacam-se BGH-2143, BGH-2235, BGH-2270 e BGH-2118, os quais compuseram os grupos 23 e 24 . Esses acessos apresentam valores reduzidos de $\mathrm{B}_{3}$ e AACPD (Tabela 4), além de curvas com menores valores de severidade da pinta-preta (Figura 2); portanto, podem ser considerados potenciais fontes de resistência à doença. Essas informações são importantes, uma vez que, segundo Kumar \& Srivastava (2013), poucos estudos apresentam fontes de resistência à pinta-preta dentro da espécie cultivada de tomateiro. De acordo com esses mesmos autores, a maioria dos acessos resistentes à pinta-preta pertence à espécie $S$. habrochaites.

Tabela 4. Valores médios e desvio-padrão de $\mathrm{B}_{1}, \mathrm{~B}_{3}$ e $\mathrm{AACPD}$, estimados pelo ajuste dos dados de severidade da pinta-preta (Alternaria tomatophila) ao modelo logístico, para cada grupo formado por meio da análise multivariada ${ }^{(1)}$.

\begin{tabular}{|c|c|c|c|c|c|c|}
\hline Grupo & $\mathrm{B}_{1}$ & $\mathrm{DP}\left(\mathrm{B}_{1}\right)$ & $\mathrm{B}_{3}$ & $\mathrm{DP}\left(\mathrm{B}_{3}\right)$ & AACPD & $\mathrm{DP}$ (AACPD) \\
\hline 1 & 0,0001 & - & 1,3551 & - & 768,0834 & - \\
\hline 2 & 0,0084 & 0,0123 & 1,1818 & 0,0192 & 756,9584 & 37,3572 \\
\hline 3 & 0,0195 & 0,0113 & 1,0130 & 0,0488 & 707,6946 & 38,3770 \\
\hline 4 & 0,5868 & - & 0,6199 & - & 580,5834 & - \\
\hline 5 & 0,2760 & 0,0305 & 0,7256 & 0,0382 & 549,2918 & 143,7075 \\
\hline 6 & 0,2200 & 0,0029 & 0,8770 & 0,0342 & 496,0001 & 234,8774 \\
\hline 7 & 0,4096 & - & 1,0678 & - & 476,7502 & - \\
\hline 8 & 0,6411 & 0,0076 & 0,5213 & 0,0241 & 473,0419 & 48,1426 \\
\hline 9 & 0,1325 & - & 0,6728 & - & 458,3333 & - \\
\hline 10 & 0,5196 & 0,0252 & 0,4575 & 0,0288 & 436,5835 & 67,8173 \\
\hline 11 & 0,2000 & 0,0404 & 0,5641 & 0,0410 & 418,3543 & 71,2448 \\
\hline 12 & 0,0513 & 0,0468 & 0,7811 & 0,0332 & 405,2180 & 102,7263 \\
\hline 13 & 0,0594 & 0,0295 & 0,6102 & 0,0465 & 388,3948 & 100,1880 \\
\hline 14 & 0,5924 & 0,0366 & 0,3694 & 0,0429 & 387,9167 & 83,0826 \\
\hline 15 & 1,0728 & 0,0364 & 0,2728 & 0,0436 & 383,8334 & 121,5045 \\
\hline 16 & 0,6760 & 0,0286 & 0,3122 & 0,0307 & 383,3334 & 113,6706 \\
\hline 17 & 0,8065 & 0,0316 & 0,2836 & 0,0387 & 367,7500 & 83,5484 \\
\hline 18 & 0,2618 & 0,0490 & 0,4146 & 0,0425 & 357,4524 & 76,6298 \\
\hline 19 & 0,7367 & 0,0334 & 0,4164 & 0,0370 & 351,3334 & 21,5667 \\
\hline 20 & 0,3428 & - & 0,5421 & - & 337,7499 & - \\
\hline 21 & 0,4139 & 0,0407 & 0,3764 & 0,0428 & 333,9465 & 103,1736 \\
\hline 22 & 0,0035 & 0,0024 & 0,8871 & 0,0245 & 324,0417 & 44,8189 \\
\hline 23 & 0,1073 & 0,0107 & 0,4466 & 0,0413 & 293,7498 & 116,6142 \\
\hline 24 & 0,6897 & - & 0,1644 & - & 93,0835 & - \\
\hline
\end{tabular}

${ }^{(1)} \mathrm{B}_{1}$ e $\mathrm{B}_{3}$, média dos parâmetros do modelo logístico; DP, desvio-padrão dos parâmetros do modelo logístico; e AACPD, média da área abaixo da curva de progresso da doença. 
A análise do desempenho de acessos de tomateiro cultivado é extremamente útil aos programas de melhoramento da cultura. Conforme Marim et al.
(2009), a existência de acessos semelhantes às cultivares é interessante, pois, caso haja alguma característica diferencial, como a resistência a doenças,

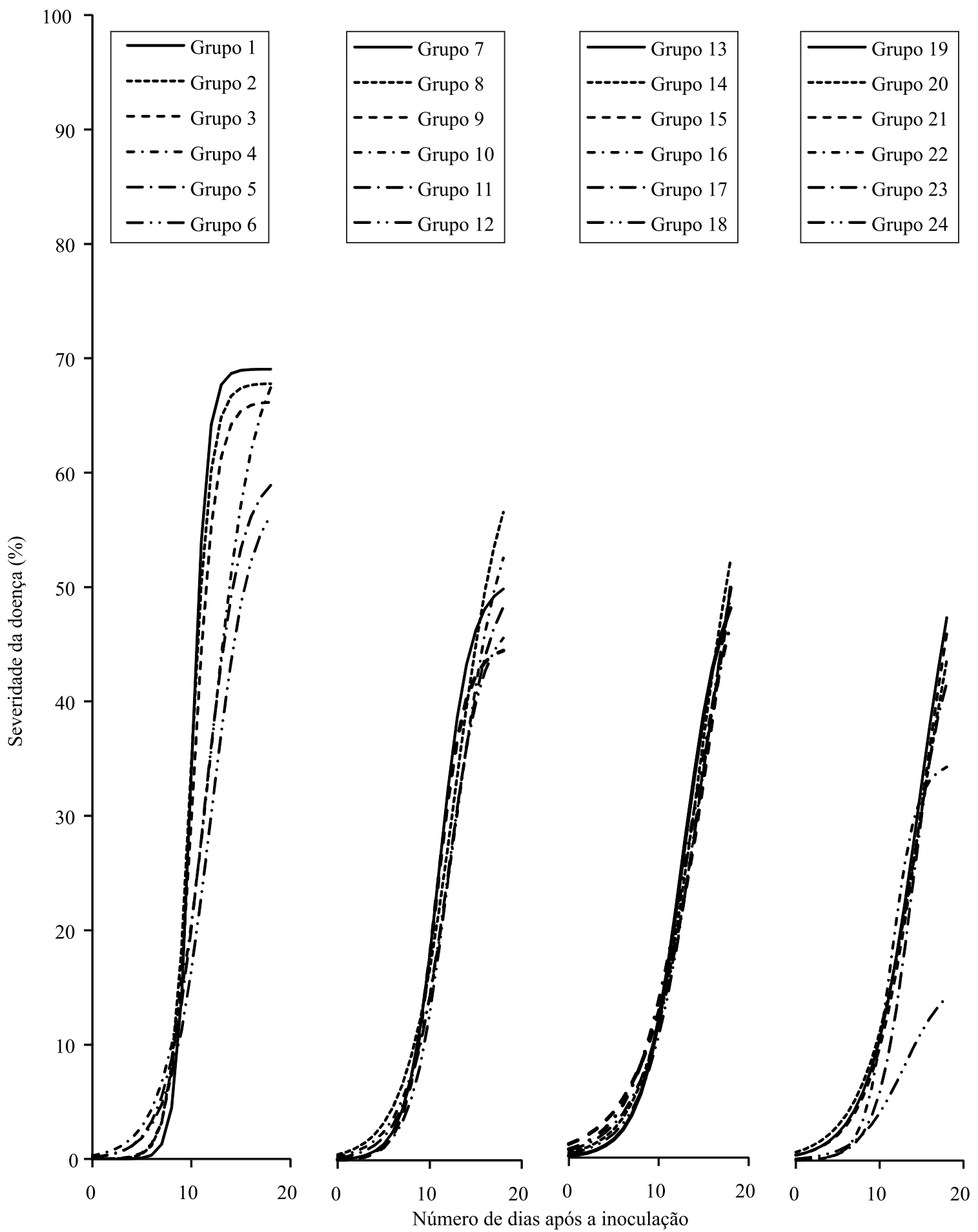

Figura 2. Curva de progresso da pinta-preta (Alternaria tomatophila) em tomateiro (Solanum lycopersicum) para cada grupo obtido via análise de agrupamento. Para melhor visualização, as 24 curvas foram divididas em quatro gráficos. 
sua inclusão nos programas de melhoramento não prejudicaria demasiadamente as demais características de interesse agronômico em função da não ocorrência de arraste gênico, comum quando genitores silvestres são utilizados.

Os quatro acessos identificados como fontes de resistência à pinta-preta são passíveis de utilização em programas de melhoramento do tomateiro e podem possibilitar a redução do progresso da doença no campo, das perdas decorrentes da doença e do número de aplicações de fungicidas para o seu controle.

\section{Conclusões}

1. A análise de agrupamento multivariada reduz o número de curvas de progresso da doença, na avaliação da resistência do tomateiro (Solanum lycoperscium) à pinta-preta (Alternaria tomatophila).

2. Os acessos BGH-2143, BGH-2235, BGH-2270 e BGH-2118, pertencentes à espécie S. lycopersicum, são potenciais fontes de resistência à pinta-preta.

\section{Agradecimentos}

À Coordenação de Aperfeiçoamento de Pessoal de Nível Superior (Capes), ao Conselho Nacional de Desenvolvimento Científico e Tecnológico $(\mathrm{CNPq})$ e à Fundação de Amparo à Pesquisa do Estado de Minas Gerais (Fapemig), pela concessão de bolsas e pelo apoio financeiro; e à Universidade Federal de Viçosa (UFV), pelo apoio na realização do trabalho.

\section{Referências}

AZEVEDO, C.F.; SILVA, F.F. e; RIBEIRO, N.B.; SILVA, D.J.H. da; CECON, P.R.; BARILI, L.D.; PINHEIRO, V.R. Classificação multivariada de curvas de progresso da requeima do tomateiro entre acessos do Banco de Germaplasma de Hortaliças da UFV. Ciência Rural, v.42, p.414-417, 2012. DOI: 10.1590/ S0103-84782012000300005.

CAMPBELL, C.L.; MADDEN, L.V. (Ed.). Introduction to plant disease epidemiology. New York: Wiley, 1990. 532p.

CARDOSO, C.R. Agressividade de Alternaria tomatophila, A. grandis e A. solani em batateira e tomateiro. 2010. 43p. Dissertação (Mestrado) - Universidade Federal de Viçosa, Viçosa.

CATÃO, H.C.R.M.; SALES, N. de L.P.; AZEVEDO, D.M.Q.; FLÁVIO, N.S.D. da S.; MENEZES, J.B. de C.; BARBOSA, L.V.; MARTINEZ, R.A.S. Fungicides and alternative products in the mycelial growth and germination control of Alternaria tomatophila. Idesia, v.31, p.21-28, 2013. DOI: 10.4067/ S0718-34292013000300004.
CECON, P.R.; SILVA, F.F. e; FERREIRA, A.; FERRÃO, R.G.; CARNEIRO, A.P.S.; DETMANN, E.; FARIA, P.N.; MORAIS, T.S. da $\mathrm{S}$. Análise de medidas repetidas na avaliação de clones de café 'Conilon'. Pesquisa Agropecuária Brasileira, v.43, p.1171-1176, 2008. DOI: 10.1590/S0100-204X2008000900011.

CRUZ, C.D.; CARNEIRO, P.C.S.; REGAZZI, A.J. Modelos biométricos aplicados ao melhoramento genético. 3.ed. Viçosa: Ed. da UFV, 2014. v.2, 668p.

FIORINI, C.V.A.; SILVA, D.J.H. da; SILVA, F.F. e; MIZUBUTI, E.S.G.; ALVES, D.P.; CARDOSO, T. de S. Agrupamento de curvas de progresso de requeima em tomateiro originado de cruzamento interespecífico. Pesquisa Agropecuária Brasileira, v.45, p.1095-1101, 2010. DOI: 10.1590/S0100-204X2010001000007.

GRIGOLLI, J.F.J.; KUBOTA, M.M.; ALVES, D.P.; RODRIGUES, G.B.; CARDOSO, C.R.; D.J.H.; SILVA, F.F.; MIZUBUTI, E.S.G. Characterization of tomato accessions for resistance to early blight. Crop Breeding and Applied Biotechnology, v.11, p.174-180, 2011. DOI: $10.1590 /$ S1984-70332011000200010.

KUMAR, S.; SRIVASTAVA, K. Screening of tomato genotypes against early blight (Alternaria solani) under field condition. The Bioscan, v.8, p.189-193, 2013.

LIU, Y.; LI, Z.; XIONG, H.; GAO, X.; WU, J.; WU, S. Understanding and enhancement of internal clustering validation measures. IEEE Transactions on Cybernetics, v.43, p.982-993, 2013. DOI: 10.1109/TSMCB.2012.2220543.

MAIA, E.; SIQUEIRA, D.L. de; SILVA, F.F. e; PETERNELLI, L.A.; SALOMÃO, L.C.C. Método de comparação de modelos de regressão não-lineares em bananeiras. Ciência Rural, v.39, p.1380-1386, 2009. DOI: 10.1590/S0103-84782009000500012.

MARIM, B.G.; SILVA, D.J.H.da; CARNEIRO, P.C.S.; MIRANDA, G.V.; MATTEDI, A.P.; CALIMAN, F.R.B. Variabilidade genética e importância relativa de caracteres em acessos de germoplasma de tomateiro. Pesquisa Agropecuária Brasileira, v.44, p.1283-1290, 2009. DOI: 10.1590/S0100-204X2009001000011.

NUTTER, J.R.F.W. Disease severity assessment training. In: FRANCL, L.J.; NEHER, D.A. (Ed.). Exercises in plant disease epidemiology. St. Paul: American Phytopathological Society, 1997. p.1-7.

PAULA, R.S. de; OLIVEIRA, W.F. de. Resistência de tomateiro (Lycopersicon esculentum) ao patógeno Alternaria solani. Pesquisa Agropecuária Tropical, v.33, p.89-95, 2003.

PIMENTEL-GOMES, F. Curso de estatística experimental. 15.ed. São Paulo: Fealq, 2009. 451p.

PUIATTI, G.A.; CECON, P.R.; NASCIMENTO, M.; PUIATTI, M.; FINGER, F.L.; SILVA, A.R. da; NASCIMENTO, A.C.C. Análise de agrupamento em seleção de modelos de regressão não lineares para descrever o acúmulo de matéria seca em plantas de alho. Revista Brasileira de Biometria, v.31, p.337-351, 2013.

QUINTANA, A.C. Agrianual 2013: anuário da agricultura brasileira. Campo Grande: FNP Consultoria e Comércio, 2013. 480 p.

SAS INSTITUTE. Statistical analysis system user's guide. Version 9.0. Cary: SAS Institute, 2002. 513p. 
SILVEIRA, F.G. da; SILVA, F.F. e; CARNEIRO, P.L.S.; MALHADO, C.H.M.; MUNIZ, J.A. Análise de agrupamento na seleção de modelos de regressão não-lineares para curvas de crescimento de ovinos cruzados. Ciência Rural, v.41, p.692-698, 2011. DOI: 10.1590/S0103-84782011000400024.
VALE, F.X.R.; JESUS JUNIOR, W.C.; RODRIGUES, F.A.; COSTA, H.; SOUZA, C.A. Manejo de doenças fúngicas em tomateiro. In: SILVA, D.J.H.; VALE, F.X.R. (Ed.). Tomate: tecnologia de produção. Visconde do Rio Branco: Suprema, 2007. v.1, p.159-198.

Recebido em 17 de junho de 2014 e aprovado em 19 de janeiro de 2015 